\title{
Adjunctive Lurasidone Suppresses Food Intake and Weight Gain Associated with Olanzapine Administration in Rats
}

\author{
Gavin P. Reynolds ${ }^{1}$, Caroline F. Dalton ${ }^{1}$, William Watrimez ${ }^{2}$, Joshua Jackson ${ }^{2}$, Michael K. Harte ${ }^{2}$ \\ ${ }^{1}$ Biomolecular Sciences Research Centre, Sheffield Hallam University, Sheffield, ${ }^{2}$ Division of Pharmacy and Optometry, School of Health \\ Sciences, Manchester, UK
}

\begin{abstract}
Objective: Lurasidone is an antipsychotic drug that shows a relative lack of weight gain common to many antipsychotics. Aripiprazole and ziprasidone also show little weight gain and can reduce olanzapine-induced food intake and weight gain in animals, paralleling some clinical findings. We hypothesized that lurasidone would have similar actions. Methods: Female Lister-hooded rats received intraperitoneal injection either $2 \times$ vehicle (saline), lurasidone $(3 \mathrm{mg} / \mathrm{kg})$ and vehicle, olanzapine $(1 \mathrm{mg} / \mathrm{kg}$ ) and vehicle, or olanzapine and lurasidone. Following drug administration food intake was measured for $60 \mathrm{~min}$. A further series of rats underwent a seven-day regime of once-daily administration of the above doses and free access to food and water. Weight gain over the course of the study was monitored.

Results: Olanzapine induced a significant increase in food intake while lurasidone showed no significant effect. Co-administration of lurasidone with olanzapine suppressed the increase in food intake. Repeated dosing showed an increase in body weight after seven days with olanzapine, and no significant effect observed with lurasidone, while repeated administration of lurasidone with olanzapine reduced the effect of olanzapine on the increase in body weight.

Conclusion: These findings support our hypotheses in that lurasidone, in addition to a lack of effect on acute food intake and short term weight gain, can reduce olanzapine-induced food intake and weight gain in rats. This indicates the drug to have an active anti-hyperphagic mechanism, rather than solely the absence of a drug-induced weight gain that is such a severe limitation of drugs such as olanzapine.
\end{abstract}

KEY WORDS: Antipsychotic agents; Eating; Weight gain; Lurasidone hydrochloride; Olanzapine.

\section{INTRODUCTION}

Many antipsychotic drugs demonstrate adverse effects on body weight and metabolic syndrome, primarily a clinically significant weight gain emerging within 6 weeks of initial treatment in many subjects. ${ }^{1)}$ Among the commonly-prescribed antipsychotic drugs, clozapine and olanzapine exhibit the greatest weight gain. However antipsychotic-induced weight gain is not restricted to second-generation drugs; furthermore, some antipsychotics induce negligible weight gain. A recent multi-comparison meta-analysis indicated that only haloperidol, ziprasi-

Received: February 22, 2018 / Revised: April 2, 2018

Accepted: April 3, 2018

Address for correspondence: Gavin P. Reynolds, PhD

Biomolecular Sciences Research Centre, Sheffield Hallam

University, Howard St, Sheffield S1 1WB., UK

Tel: +44-114-225-5555, Fax: +44-114-225-4449

E-mail: gavin.reynolds@hotmail.com

ORCID: https://orcid.org/0000-0001-9026-7726 done, and lurasidone did not differ from placebo levels of weight gain, while these drugs, along with aripiprazole, had significantly less weight gain than quetiapine and risperidone, which in turn show significantly less weight gain than olanzapine. ${ }^{2)}$

We and others have demonstrated that the weight gain, which reflects increased food intake, occurring in patients receiving olanzapine can be reliably modelled in the rat in acute effects on food intake and satiety and both short and longer term effects on body weight. ${ }^{3,4)}$ We have also found that ziprasidone can reverse the hyperphagic effect of olanzapine. ${ }^{3)}$ This indicates that ziprasidone has a pharmacological action that goes beyond a lack of olanzapine-like hyperphagia to include an additional protective component. We see the same effect with aripiprazole $^{5)}$ and have proposed that a partial agonist/antagonist action at the 5-HT1A receptor of ziprasidone and aripiprazole may be one mechanism that could contribute to

(c) This is an Open-Access article distributed under the terms of the Creative Commons Attribution Non-Commercial License (http://creativecommons.org/licenses/by-nc/4.0) which permits unrestricted non-commercial use, distribution, and reproduction in any medium, provided the original work is properly cited. 
these findings, although other pharmacological effects may be important. ${ }^{6)}$ The results have implications in the clinic: it has been observed that aripiprazole can ameliorate weight gain and some other metabolic changes in patients receiving olanzapine ${ }^{7)}$ or clozapine. ${ }^{8)}$

The current study was to determine whether this protective mechanism against olanzapine-induced food intake and weight gain extends to lurasidone, given that this drug too has effects at the 5-HT1A receptor and in clinical trials has been shown to be relatively free of a propensity for weight gain. ${ }^{2)}$ We specifically tested the hypotheses that co-administration of lurasidone would suppress the acute increase in food intake following olanzapine administration to rats, and would also suppress the short term (one week) weight gain seen following daily administration of olanzapine.

\section{METHODS}

Adult female Lister hooded rats housed under standard laboratory conditions with a 12-hour cycle (lights on at 7:00) were habituated to the experimental procedure in individual cages with a palatable mash food preparation ${ }^{5}$ for 4 days prior to the test day. The day before the test day all animals received a saline injection prior to injections on the test day. On the test day all animals were put in the test cages and given 30 minutes access to the mash (initial food intake). They were then removed and given the appropriate injections; four groups of six animals received either $2 \times$ vehicle (saline), lurasidone $(3 \mathrm{mg} / \mathrm{kg}$, intraperitoneal injection) and vehicle, olanzapine $(1 \mathrm{mg} / \mathrm{kg}$,

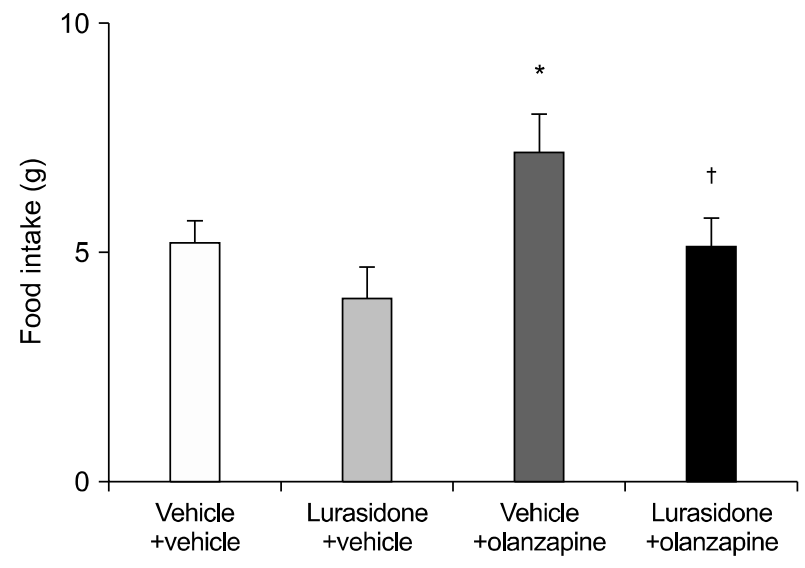

Fig. 1. Food intake over 60 minutes following drug administration. ${ }^{*} p<0.05$ vs. vehicle; ${ }^{\dagger} p<0.05$ vs. olanzapine. intraperitoneal injection) and vehicle, or olanzapine and lurasidone. Drug doses were chosen based on our prior experience with olanzapine in inducing weight gain ${ }^{4)}$ and the effective dose of lurasidone in an animal model of antipsychotic efficacy. ${ }^{9}$ Following drug administration all animals were returned to the cages with the food for a further 60 minutes (final food intake). Weight of initial and final food intake was measured.

A further series of rats underwent a seven-day dosing regime in the above groups of six animals each, receiving once-daily administration of the above doses. Animals had free access to food and water. Prior to each dose the animals were weighed, and weight gain over the course of the study was monitored. All experiments were performed according to the Animals (Scientific Procedures) Act 1986, and with approval from the University of Manchester Research Ethics Committee.

Initial statistical analysis was undertaken by analysis of variance using IBM SPSS Statistics ver. 21.0 (IBM Corp., Armonk, NY, USA) followed post-hoc by Dunnett's $t$ test.

\section{RESULTS}

The results of the initial acute study are indicated in Figure 1. Analysis of variance demonstrated a significant overall effect $(\mathrm{F}=3.818, p=0.027)$. There is a significant increase in food intake with olanzapine ( $p=0.031$ vs. vehicle) in the absence of any significant effect of lurasidone administration. Notably, co-administration of lurasidone with olanzapine suppresses the increase in food intake seen with the latter drug ( $p=0.043$ vs. olanzapine).

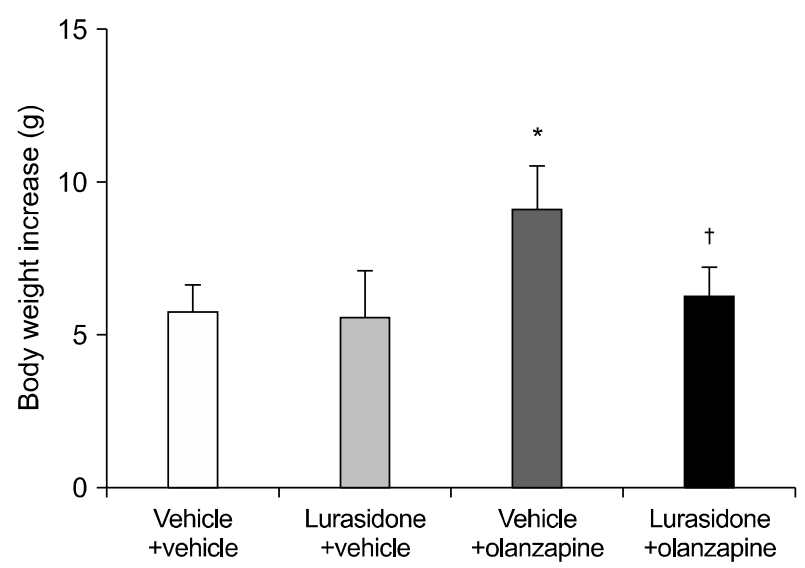

Fig. 2. Weight gain over 7 days of drug administration. ${ }^{*} p<0.05$ vs. no olanzapine; ${ }^{\dagger} p=0.10$ vs. olanzapine. 
The short term dosing study (Fig. 2) showed the same pattern of results, although the overall effect was not significant $(\mathrm{F}=1.652, p=0.209)$. Nevertheless, further exploratory analysis showed an increase in body weight over control and lurasidone animals after seven days' administration of olanzapine $(p=0.031)$, with no mean increase observed with lurasidone. Furthermore, the repeated administration of lurasidone with olanzapine showed an apparent reduction in the effect of olanzapine administration alone on the increase in body weight, although this did not achieve statistical significance $(p=$ 0.10 vs. olanzapine).

\section{DISCUSSION}

These preliminary findings clearly support our initial hypotheses in showing that lurasidone, in addition to demonstrating a lack of effect on acute food intake in clear contrast to olanzapine, also demonstrates a protective effect on olanzapine-induced food intake in rats. Similar effects were observed in the short-term weight gain study, although the findings did not achieve statistical significance. This apparently protective mechanism on food intake has also been demonstrated for aripiprazole and ziprasidone, indicating that the three drugs have an active anti-hyperphagic mechanism, rather than solely the absence of the pharmacological propensity for weight gain and which is such a severe limitation of drugs such as olanzapine.

This study is both small and short-term and thus would benefit from replication and extension in both size and time course. The weaker effects on weight gain that we observed reflected the presence of a single animal in the olanzapine group that showed minimal ( $3 \mathrm{~g}$ ) weight gain in comparison to a range of 7 to $12 \mathrm{~g}$ for the other rats receiving olanzapine. Nevertheless these findings indicate the value of investigating whether there might be a beneficial effect of adjunctive lurasidone in the amelioration and/or attenuation of antipsychotic-induced weight gain, an effect that is clinically well established for aripiprazole. $^{10)}$ We have used female animals since in our experience they more readily demonstrate antipsychotic-induced weight gain than males; this observation has been discussed previously. ${ }^{4)}$

Current understanding of the pharmacological mechanism(s) underlying weight gain following antipsychotic drug treatment is far from complete. We found olanzapine-induced weight gain can be effectively modelled in the short term by a combination of a D2 antagonist (haloperidol) and a 5-HT2C inverse agonist, but not by a D2 and histamine $\mathrm{H} 1$ antagonist combination. ${ }^{4)}$ Thus one contributory process may be the action of drugs on hypothalamic neurotransmitter receptors including, but not necessarily exclusively, the $5-\mathrm{HT} 2 \mathrm{C}$ receptor, which is likely to disrupt signalling from hormones such as leptin. ${ }^{6)}$ This concept provides a good model for understanding some effects of drugs with high metabolic risk such as olanzapine, although it may not fully account for the intermediate effects of some other antipsychotic drugs ${ }^{2)}$ for which other receptors including histamine $\mathrm{H} 1$ may be important. Certainly serotonin neurotransmission has an important role in the regulation of food intake and body weight and thus drugs acting at $5 \mathrm{HT}$ receptors may influence these processes. One other receptor important in eating behaviour is the $5-\mathrm{HT} 1 \mathrm{~A}$ site ${ }^{11)}$; this can modulate presynaptic neuronal activity as well as having postsynaptic action. 5-HT1 A and 5-HT2C receptors have opposite effects on body weight, and the 5-HT1A partial (ant)agonism of lurasidone, as we have suggested for ziprasidone and aripiprazole, ${ }^{6)}$ may contribute to inhibiting orexigenic effects of antipsychotics, such as olanzapine and clozapine, that have strong 5-HT2C inverse agonism. ${ }^{12)}$ This hypothesis has yet to be tested, however.

\section{REFERENCES}

1. Zhang ZJ, Yao ZJ, Liu W, Fang Q, Reynolds GP. Effects of antipsychotics on fat deposition and changes in leptin and insulin levels. Magnetic resonance imaging study of previously untreated people with schizophrenia. Br J Psychiatry 2004;184: 58-62.

2. Leucht S, Cipriani A, Spineli L, Mavridis D, Orey D, Richter F, et al. Comparative efficacy and tolerability of 15 antipsychotic drugs in schizophrenia: a multiple-treatments meta-analysis. Lancet 2013;382:951-962.

3. Kirk SL, Neill JC, Jones DN, Reynolds GP. Ziprasidone suppresses olanzapine-induced increases in ingestive behaviour in the rat. Eur J Pharmacol 2004;505:253-254.

4. Kirk SL, Glazebrook J, Grayson B, Neill JC, Reynolds GP. Olanzapine-induced weight gain in the rat: role of 5-HT2C and histamine $\mathrm{H} 1$ receptors. Psychopharmacology (Berl) 2009;207:119-125.

5. Snigdha S, Thumbi C, Reynolds GP, Neill JC. Ziprasidone and aripiprazole attenuate olanzapine-induced hyperphagia in rats. J Psychopharmacol 2008;22:567-571. 
6. Reynolds GP, Kirk SL. Metabolic side effects of antipsychotic drug treatment--pharmacological mechanisms. Pharmacol Ther 2010;125:169-179.

7. Henderson DC, Fan X, Copeland PM, Sharma B, Borba CP, Boxill R, et al. Aripiprazole added to overweight and obese olanzapine-treated schizophrenia patients. / Clin Psychopharmacol 2009;29:165-169.

8. Fleischhacker WW, Heikkinen ME, Olié JP, Landsberg W, Dewaele P, McQuade RD, et al. Effects of adjunctive treatment with aripiprazole on body weight and clinical efficacy in schizophrenia patients treated with clozapine: a randomized, double-blind, placebo-controlled trial. Int J Neuropsychopharmacol 2010;13:1115-1125.

9. Kołaczkowski M, Mierzejewski P, Bienkowski P, Wesołowska A, Newman-Tancredi A. Antipsychotic, antidepressant, and cognitive-impairment properties of antipsychotics: rat profile and implications for behavioral and psychological symptoms of dementia. Naunyn Schmiedebergs Arch Pharmacol 2014; 387:545-557.

10. Mizuno Y, Suzuki T, Nakagawa A, Yoshida K, Mimura M, Fleischhacker WW, et al. Pharmacological strategies to counteract antipsychotic-induced weight gain and metabolic adverse effects in schizophrenia: a systematic review and metaanalysis. Schizophr Bull 2014;40:1385-1403.

11. Dourish CT, Hutson PH, Curzon G. Low doses of the putative serotonin agonist 8-hydroxy-2-(di-n-propylamino) tetralin (8-OH-DPAT) elicit feeding in the rat. Psychopharmacology (Berl) 1985;86:197-204.

12. Herrick-Davis K, Grinde E, Teitler M. Inverse agonist activity of atypical antipsychotic drugs at human 5-hydroxytryptamine2C receptors. J Pharmacol Exp Ther 2000;295:226-232. 